1 Running head: Sub-movements in Aiming Tasks

\title{
2 Are Sub-Movements Induced Visually in Discrete Aiming Tasks?
}

4 Tsung-Yu Hsieh ${ }^{1,2,3}$, Matheus M. Pacheco ${ }^{4,5}$, Yeou-Teh Liu ${ }^{6 *}$ and Karl M. Newell ${ }^{7}$

$6 \quad{ }^{1}$ Department of Physical Education, Fu Jen Catholic University - New Taipei, Taiwan

$7 \quad{ }^{2}$ Research and Development Center for Physical Education, Health and Information

8 Technology, Fu Jen Catholic University - New Taipei, Taiwan

$9{ }^{3}$ Physical Education Office, Fu Jen Catholic University - New Taipei, Taiwan

$10{ }^{4}$ School of Physical Education and Sport at Ribeirão Preto, University of São Paulo -

11 Ribeirão Preto, Brazil

$12{ }^{5}$ Movement Control \& Neuroplasticity Research Group, KU Leuven - Leuven, Belgium.

$13{ }^{6}$ Department of Athletic Performance, National Taiwan Normal University - Taipei, Taiwan.

$14{ }^{7}$ Department of Kinesiology, University of Georgia - Athens, USA.

$16{ }^{*}$ Corresponding author:

17 Yeou-Teh Liu

18 Department of Athletic Performance, National Taiwan Normal University, 88, Ting-Zhou

19 Road Section 4, Taipei 116, Taiwan.

20 Email: yeouteh@ntnu.edu.tw 


\section{Abstract}

There is a long-held view that discrete movements aimed to a target are composed of

3 a sequence of movement units (sub-movements) that have different roles in motor control

4 (e.g., initial impulse, error correction and movement termination) depending on the task

5 constraints (e.g., spatial-temporal requirements). Here we report findings from the

6 manipulation of vision/no-vision on the prevalence and type of sub-movements in discrete

7 movement tasks over a range of space-time task criteria. The presence of vison resulted in

8 longer movement times compared to the no-vision counterpart in time-matching tasks. A

9 similar vision effect was observed in the highest Index of Difficulty for time-minimization

10 tasks. Conditions that resulted/required longer movement times demonstrated more pre-

11 velocity-peak and post-velocity-peak types of sub-movements whereas short movement times

12 increased the likelihood of overshooting sub-movements. The present study results are

13 consistent with the idea that movement time is the variable associated with changes in sub-

14 movement profiles.

15 Keywords: motor control, goal directed task, space-time constraint, Fitts law, feedback, 16 visual corrections 


\section{Introduction}

2 Since Woodworth (1899), there has been a long-held view that discrete movements

3 aimed to a target are composed of smaller units of movement (sub-movements) that have

4 different roles in movement production. Woodworth proposed that there was an initial large-

5 amplitude sub-movement (impulse) in the direction of the target to change stylus location in a

6 short time period followed by a secondary sub-movement performing a correction to the first

7 initial impulse. These sub-movements have generally been characterized through "zero-

8 crossings" in the velocity, acceleration or jerk profiles of movement over time (see, for

9 instance, Meyer et al., 1990) and have been interpreted as primarily under visual control

10 (Carlton, 1979; Crossman \& Goodeve, 1983; Keele \& Posner, 1968). This theoretical

11 approach has provided experimental evidence for sub-movements and visually based

12 intermittent models of movement control (Crossman \& Goodeve, 1983; Elliott et al., 2001,

13 2017; Meyer et al., 1990; Milner, 1992).

14 The literature has diverged, however, on the interpretation of what would be the relevant

15 sub-movements in movements aimed to a target. Elliott and colleagues (see Elliott et al.,

16 2001, 2017), on the one hand, have provided evidence on the error correction account of

17 Woodworth (1899) and have expanded this view in a model that includes changes with

18 practice in aiming tasks. Dounskaia and colleagues (e.g., Dounskaia et al., 2005, 2009;

19 Fradet, Lee, \& Dounskaia, 2008), on the other hand, have shown that the zero-crossings in

20 kinematic profiles can emerge from mechanisms other than visually-based movement

21 corrections; namely, motion termination or slow movement control (see also Plamondon \&

22 Alimni, 1997 for other non-corrective sources of sub-movements). The former refers to sub-

23 movements generated when the arm reaches the target with zero velocity but non-zero

24 acceleration eliciting "dwelling on target" while the latter refers to irregular fluctuations that 
1 occur commonly in slow/long duration movements (Fradet et al., 2008; Milner \& Ijaz, 1990;

2 Wisleder \& Dounskaia, 2007). Evidence for these "mechanically-based" sub-movements

3 comes from comparisons between task conditions in which corrective sub-movements were

4 deemed not necessary (i.e., reciprocal and "passing-through" aiming paradigms, see Fradet et 5 al., 2008).

6 In our view, the experimental approaches to untangle the role of vision in the control of 7 sub-movements have not been sufficient to reveal the involvement of visual control. First, the 8 general interpretations of the source of sub-movements are not mutually exclusive and not 9 differentiable through kinematic profiles. Corrections and mechanical oscillations will both 10 lead to zero-crossing patterns in the kinematic profile of the aiming trajectory and both can 11 occur in any given movement. Second, the reciprocal and passing-through paradigms were used with the assumption that they require no corrective movements (submovements in the

13 latter were analyzed after the target was passed). Nevertheless, these assumptions, especially

14 for the reciprocal Fitts' paradigm, might not hold. Third, the use of conditions that

15 manipulate directly visual control in the assessment of the role of vision in sub-movements

16 and error correction has not been implemented sufficiently.

17 In Fitts' original reciprocal paradigm (Fitts, 1954), the instructional emphasis was on 18 accuracy and $95 \%$ or more was obtained as participants would exploit the allowed tolerance 19 during the task. Individuals could miss the target and might need to perform corrections on 20 some trials. In fact, it was commonplace to require adjustments in the accuracy achieved for 21 Fitts' law to hold when individuals did not achieve 95\% accuracy (see, for instance, Welford et al., 1969). Thus, the reciprocal paradigm cannot be considered to result in no visually-

23 induced corrections. A proper test of sub-movements-as-more-than-just-corrections should be 24 performed on conditions where corrections are not actually made. Also, for the passing- 
1 through paradigm, it is questionable as to what degree the findings from this paradigm relate

2 to sub-movements in other movement aiming paradigms.

3 One way to avoid the challenges of isolating task influences altogether would be to

4 compare conditions where visual corrections either are or are not possible. That is, one must

5 be sure that, in a given condition, online corrections are not possible and then compare these

6 with those conditions which corrections naturally emerge. The assumption in these aiming

7 paradigms is that if there are corrections, they are mainly visually-driven (Carlton, 1979;

8 Crossman \& Goodeve, 1983; Chua \& Elliott, 1993; Keele \& Posner, 1968; Woodworth,

9 1899). Thus, a fundamental way to verify the existence of non-corrective sub-movements is

10 to compare aiming in situations that allow and do not allow the availability of visual

11 information during movement execution.

12 The present experiment was set-up to examine the presence of visually induced

13 corrections in discrete aiming movements over a range of spatial and temporal constraints.

14 We used the identifying movement correction protocol of Chua and Elliott (1993) to detect

15 the presence of the 4 types of movement corrections (Hsieh et al., 2017; 2019): namely,

16 initial primary impulse, pre-peak velocity, post-peak velocity and overshooting (Figure 1). To

17 examine a broad range of discrete task conditions, the experiment used two different discrete

18 aiming paradigms (time-minimization [Fitts \& Peterson, 1964] or spatially constrained task

19 [cf. Meyer et al., 1988], and time-matching [Schmidt et al., 1979] or temporally constrained

20 task [cf. Meyer et al., 1988]) both with and without vision. The inclusion of no-vision

21 conditions allowed us to observe whether sub-movement occurrences are indeed dependent on visual information. If sub-movements observed in the no-vision conditions are of the same number and same type as those observed in the vision conditions, this would support the view

24 that sub-movements in the typical-vision-available conditions of aiming movements are not 25 visually-based corrections. 
1 The target size in the time-minimization (Fitts' law) paradigm was manipulated over a

2 higher than usual range of index of difficulty (ID, 2 to 8) (Fitts, 1954) to require different

3 spatial-temporal strategies inducing changes in sub-movement profiles. The movement time

4 criterion was manipulated in the time-matching paradigm to examine the effect of movement

5 velocity in sub-movement presence and type and, as in the time-minimization paradigm, to

6 test whether these emerge as corrective processes by comparing with no-vision conditions.

7 The collective of movement conditions manipulated here provided for investigation of the

8 interaction of several variables and processes in speed and accuracy that are relevant to the

9 interpretation of sub-movements as being induced by visual information in the context of a

10 particular combination of spatial-temporal task constraints.

\section{Methods}

\section{Participants}

Twenty self-reported right-handed healthy young adults (age: 20-35 yrs) were recruited

17 for the experiment. All participants provided informed written consent before participating in

18 the experiment. The Research Ethic Committee at the National Taiwan Normal University

19 approved the experimental procedures.

\section{Apparatus}

21 Line drawing movements were made on a WACOM Cintiq 27 digital tablet (Model

DTK-2700/K0-CX, 770mm $\times 465 \mathrm{~mm}$ with active surface area of $596.7 \mathrm{~mm} \times 335.6 \mathrm{~mm}$ ) by using a handheld stylus (Pro pen, Model KP-503E) with a weight of $18 \mathrm{~g}$. The digital tablet

24 monitor was connected to a PC computer (the pixel range was set at $1680 \times 1050$ ) and angled

$2515^{\circ}$ forward on the tabletop and placed in front of participants. A customized line drawing 
1 software was used to manipulate different criteria of movement time, amplitude and target

2 width in different conditions. The program also calculated the movement time and movement

3 errors (spatial and temporal errors) immediately after each trial for the participants. The

4 distance moved by the cursor represented on the screen to the actual distance moved of the

5 stylus on the tablet was 1:1. The movement kinematics were recorded by the customized

6 program for data collection at a sampling frequency of $130 \mathrm{~Hz}$. The PLATO visual occlusion

7 spectacles (Translucent Technologies Inc.) were used to occlude vision in certain conditions.

\section{Task}

9 Participants performed line drawing movements from left (home position: $2 \mathrm{~mm}$ x $2 \mathrm{~mm}$ )

10 to right and hit the target with a stylus on the digital tablet in spatially constrained and

11 temporally constrained tasks with and without visual information. In the spatial constrained

12 task, the movement amplitude was fixed at $30 \mathrm{~cm}$ and the target varied in widths $(2,16$, and

$13128 \mathrm{~mm}$ ) that led to 3 IDs (Fitts \& Peterson, 1964) that were 8, 5 and 2, respectively.

14 Participants were instructed to hit the target as fast as possible. In the temporally constrained

15 task, the imposed criteria of movement time were 300,500 , and $1200 \mathrm{~ms}$, all with $30 \mathrm{~cm}$

16 amplitude and $2 \mathrm{~mm}$ target width. Participants were instructed to move with a duration that

17 was within a $20 \%$ bandwidth of the temporal target ( $\pm 10 \%$ of the target time) and try to stop

18 as close as they can to a spatial target in both vision and no-vision conditions. All

19 participants completed 2 tasks within a day and each condition consisted of 20 trials. The

20 order of the tasks was counterbalanced among the participants and each task took about 30

21 mins to complete.

22 Procedures

23 Before the start of a trial, the participant sat on a chair at comfortable height. The 24 experimenter positioned the center of the tablet at the midline-view of the subject and 25 approximately $20 \mathrm{~cm}$ in front of the participant's body. Each participant was instructed to 
1 move from the home position (left) to the target (right) and match the designed criteria for all

2 tasks and conditions. At the beginning of a trial, the home position and a target were shown

3 on the tablet screen. The cursor ( $1 \mathrm{~mm}$ in diameter) showed up on the screen when the

4 participant picked-up the stylus and touched the tablet.

After the participant moved the cursor onto the home position and held it there for 600 ms, there was a beep sound to indicate to the participant to move once he/she was ready. This was not a reaction time task and the participants were informed that they could initiate their movements any time after the beep sound. The terminal temporal feedback in time

9 (movement time for the spatial task; signed temporal error for the temporal task) and space

10 (hit/miss for both tasks) from the respective task criterion were each displayed on the tablet immediately after participants completed a trial. On vision trials, participants knew vision would be available for the duration of movement. The cursor was always visible but there was no trace of the line drawing movement shown on the board that could be used as the concurrent feedback during the whole trial. During the no-vision trials, vision information would be occluded by a pair of liquid crystal spectacles after a beep sound until participants hit the target or stopped moving. The lens of the spectacles would re-open once the participant completed each movement trial.

For all conditions, the start of movement was determined by the movement velocity crossing above a threshold of $3 \mathrm{~mm} / \mathrm{s}$ for $30 \mathrm{~ms}$ and the end of the movement was defined when the movement velocity was below $3 \mathrm{~mm} / \mathrm{s}$ for greater than $30 \mathrm{~ms}$. The time between the start of movement and the end of movement was measured as the movement time (MT) of the trial. The stylus was to remain in contact with the tablet during the whole movement until the trial was completed. If the stylus left the tablet surface (a rare occurrence), the trial was

24 aborted and not considered for further analyses. After each trial, the participant moved the 
1 stylus back to the home position and waited for the beginning of the next trial. A 3 min break was provided to minimize boredom and fatigue after each condition.

Data Analyses

Movement Performance. There were 3 performance variables analyzed, MT, spatial error, and success rate. MT of each trial was measured as the time duration between the start of the line drawing movement to the end of the movement. Spatial error was determined as the distance between the end point of the line drawing movement and the center of the target in the direction of the line drawing movement (x-axis/ horizontal). The average MT and spatial error (absolute error, AE) over 20 trials were calculated for each condition for all participants. The success rate of each visual condition of each task was calculated for each participant by the number of successful trials divided by 20 . A successful trial for the spatially constrained tasks was identified as the trials on which the end of the movement occurred within the target. For the temporally constrained tasks, a successful trial was identified when the MT of the trial was within $90 \%$ to $110 \%$ of the target movement time.

Sub-movements. The movement trajectory was recorded in both the primary direction of movement to the target (x-axis/ horizontal) and in the orthogonal plane (y-axis/ vertical) coordinates of the stylus tip on the tablet. Only the displacement data on the x-axis were analyzed. The raw displacement data were low-pass filtered (second-order Butterworth filter forward and reverse order, cutoff frequency $5 \mathrm{~Hz}$ ). Movement velocity and acceleration were calculated as the first and second derivatives of movement displacement, respectively. Hsieh et al., 2017; 2019 and Figure 1 here). The first two types of sub-movements were determined by first identifying the reversal points, or local extrema, in the acceleration in the period between the start of the movement and the peak velocity (pre-peak) and between the peak velocity and the end of the movement (post-peak). When the magnitude between 
1 consecutive local extrema of the acceleration reached $10 \%$ of the maximum amplitude of the

2 acceleration, we considered it a significant deviation therefore a sub-movement. The third

3 type of sub-movement was overshooting which was characterized by a movement reversal

4 (negative velocity). For this sub-movement, the absolute amplitude of negative velocity had

5 to reach values above $10 \mathrm{~mm} / \mathrm{s}$. We did not consider undershooting sub-movement type from

6 Chua and Elliott (1993) based on the observation that there was hardly any occurrence in

7 these tasks (Hsieh et at., 2017; 2019).

8 Each movement trial had at least one sub-movement (primary-only). When the pre-peak,

9 post-peak, or overshooting types of sub-movements were identified in a trial, the number of

10 each type of the sub-movement would be added to the primary sub-movement. For example,

11 if one overshooting type of sub-movement was identified in a trial, the total number of sub-

12 movements for that trial would be 2 . The number of each type of sub-movement and total

13 number of sub-movements within a trial were determined for each participant. In addition,

14 the proportion of each type of sub-movement was computed for each condition as the number

15 of trials that contained the specific type of sub-movement divided by the number of trials

16 performed in this condition (20).

\section{Statistical Analyses}

18 To understand the effect of vision per movement goal condition in each task, we

19 performed 2 (visual conditions) X 3 (movement goals) repeated measure ANOVAs to

20 examine the success rate, MT, AE, average number of sub-movements within a trial, and the

21 proportion of each type of sub-movement. The three movement goals were ID2/ 300ms (fast),

22 ID5/ $500 \mathrm{~ms}$ (moderate), ID8/ $1200 \mathrm{~ms}$ (slow), respectively for spatially and temporally

23 constrained tasks. The Greenhouse-Geisser method was used to correct for violations of

24 sphericity and post-hoc comparisons were adjusted using Bonferroni correction, with partial

25 eta square $\left(\eta_{\mathrm{p}}^{2}\right)$ revealing the effect size. The significant level was set at $\alpha=.05$. We used 
1 Friedman's ANOVAs to examine those cases where the normal distribution assumption was

2 not fulfilled. The effect sizes were Kendal's W. Paired Wilcoxon tests were used as

3 nonparametric post-hoc tests.

4

\section{Results}

6

7 Performance

Success Rate. We first compared the success rate between vision and no-vision across the three movement goals [fast-no-vision: ID2 (309.3 $\pm 41.4 \mathrm{~ms}) / 300 \mathrm{~ms}(328.0 \pm 18.8 \mathrm{~ms})$, fastvision: ID2 (314.3 $\pm 34.5 \mathrm{~ms}) / 300 \mathrm{~ms}(345.9 \pm 29.0 \mathrm{~ms})$, moderate-no-vision: ID5 (399.6 $\pm 98.2 \mathrm{~ms}) / 500 \mathrm{~ms}(503.0 \pm 19.5 \mathrm{~ms})$, moderate-vision: ID5 $(390.8 \pm 65.9 \mathrm{~ms}) / 500 \mathrm{~ms}$ (518.3 $\pm 16.4 \mathrm{~ms})$, slow-no-vision: ID8 (597.5 $\pm 219.6 \mathrm{~ms}) / 1200 \mathrm{~ms}(1155.6 \pm 55.7 \mathrm{~ms})$, and slowvision: ID8 $(1040.0 \pm 306.3 \mathrm{~ms}) / 1200 \mathrm{~ms}(1207.8 \pm 54.3 \mathrm{~ms})]$ for the spatially constrained tasks and temporally constrained tasks. Figures $2 \mathrm{a}$ and $2 \mathrm{~b}$ show the success rate for spatially and temporally constrained tasks in each visual condition.

The ANOVA result for the spatially constrained tasks showed a significant movement goal effect, $F(2,38)=269.09, p<.001, \eta_{p}^{2}=.93$, significant visual effect, $F(1,19)=82.34, p$

$19<.001, \eta_{p}{ }^{2}=.81$, and significant interaction effect, $F(2,38)=44.41, p<.001, \eta_{p}{ }^{2}=.70$. The simple main effect of movement goal for both vision and no-vision conditions showed significantly higher success rate for the fast movement than the moderate and slow

22 movements, and significantly higher success rate for the moderate movement than the slow

23 movement condition $(p \mathrm{~s}<.001)$. The visual condition had different effect for the three

24 movement goal conditions where vision conditions had higher success rates than the no- 
1 vision conditions for the moderate and slow movement conditions $(p \mathrm{~s}<.01)$ but no

2 significant difference was found for the fast movements $(p=.16)$.

3 The similar ANOVA result was found for the temporally constrained tasks, where the 4 movement goal effect, $F(2,38)=16.55, p<.001, \eta_{p}{ }^{2}=.47$, visual effect, $F(1,19)=8.80, p$

$5<.01, \eta_{p}{ }^{2}=.32$, and the interaction effect, $F(2,38)=6.16, p<.01, \eta_{p}{ }^{2}=.25$, all reached

6 significant level. The simple main effect, however, showed a different trend from the 7 spatially constrained tasks. For the simple main effect of the movement goals, no significant

8 differences were found for the no-vision condition and between the moderate and slow

9 movement of the vision condition ( $p \mathrm{~s}>.2)$, only the fast movement goal of the vision

10 condition had significantly lower success rate than both the moderate and slow movements of

11 the vision condition $(p s<.01)$. For the simple main effect of vision, the only significant

12 difference was found for the fast movement where the vision condition had significantly

13 lower success rate than the no-vision condition $(p<.01)$.

Movement time. Figures $2 \mathrm{c}$ and $2 \mathrm{~d}$ show MT for each of the vision and no-vision conditions as a function of movement goals (for each task). For the spatially constrained tasks, the Friedman's ANOVA showed a significant effect for no-vision condition, $\chi^{2}(2)=$ 32.4, $p<.001, W=.81$, and vision condition, $\chi^{2}(2)=40, p<.001, W=1$, across different movement goals. Post hoc analysis with Wilcoxon tests showed that, for both visual conditions, the MT of the fast movement was shorter than those of the moderate and slow movements, and MT of moderate movement was shorter than that of the slow movement ( $p s$ $24<.001)$. For MT of vision and no-vision within the same movement goals, the results from the Wilcoxon comparisons showed that the only significant difference was found in the slow 
1 movement condition, where MT of vision was longer than that of no-vision $(Z=-3.88, p$

$2<.001)$.

For the temporally constrained tasks, the ANOVA showed that the main effect of movement goal was significant, $F(1.41,26.70)=6007.60, p<.001, \eta_{p}^{2}=1.0$, where the fast movement had a shorter MT than the other two conditions, and the moderate movement also

7 had a shorter MT than the slow movement. The effect of visual condition also reached a

8 significant level, $F(1,19)=26.91, p<.001, \eta_{p}{ }^{2}=.59$, where MT of no-vision was

9 significantly shorter than that of vision condition $(p<.05)$. The interaction between

10 movement goals and visual conditions did not reach a significant level, $F(1.16,22)=2.21, p$

$11=.15, \eta_{p}^{2}=.10$.

Spatial Error. Figures 2e and 2f show AE for each of the vision and no-vision conditions as a function of movement goals (for each task). For spatially constrained tasks, the Friedman's ANOVA showed a significant movement goal effect for no-vision condition, $\chi^{2}(2)=27.1, p<.001, W=.68$. Post hoc analysis showed that the fast movement had larger error than the moderate and slow movements $(p s<.001)$. For vision condition, there was also a significant movement goal effect, $\chi^{2}(2)=40, p<.001, W=1$. Post hoc tests showed the similar trend as in no-vision condition where the fast movement had larger error than the moderate and slow movements, and the moderate movement also had larger error than the slow movement $(p s<.001)$. Moreover, the results of the Wilcoxon comparisons showed significant visual effect for all movement goals: fast movement, $\mathrm{Z}=-2.84, p=.005$, moderate movement, $\mathrm{Z}=-3.73, p<.001$, and slow movement, $\mathrm{Z}=-3.92, p<.001$, where vision condition had smaller error than no-vision condition for the moderate and slow movements, but larger error was found in the vision condition of the fast movement than the no-vision condition. 
For temporally constrained tasks, the Friedman's ANOVA showed no significant

2 movement goal effect for no-vision condition, $\chi^{2}(2)=4.3, p=.12, W=.11$. For vision

3 condition, there was a significant movement goal effect, $\chi^{2}(2)=40, p<.001, W=1$. Post hoc

4 tests showed that the fast movement had larger error than the moderate and slow movements,

5 and the moderate movement had larger error than the slow movement $(p s<.001)$. Moreover,

6 the results of the Wilcoxon comparisons showed significantly smaller errors for the vision

7 than no-vision conditions for the fast, $\mathrm{Z}=-2.80, p=.005$, the moderate, $\mathrm{Z}=-3.77, p<.001$,

8 and the slow, $\mathrm{Z}=-3.92, p<.001$, movements.

\section{Sub-Movements}

10 Number of Sub-Movements. Figures 3a and 3b show the average number of sub-

11 movements in a trial for vision and no-vision as a function of movement goals. Because there

12 would be at least one sub-movement (primary-only) in a trial, any additional number of sub-

13 movements in a trial would be from pre-peak, post-peak, or overshooting types of sub-

14 movements.

15 For spatially constrained tasks, the result from Friedman's ANOVA for no-vision

16 condition showed a significant movement goal effect, $\chi^{2}(2)=7.75, p=.02, W=.19$, where

17 the number of sub-movements from the fast movement condition was greater than that from

18 the moderate movement condition $(p<.05)$. For vision condition, movement goal effect also

19 reached a significant level, $\chi^{2}(2)=24.72, p<.001, W=.62$. The post hoc comparisons

20 showed that the number of sub-movements from the slow movement was greater than those

21 from the fast and moderate movements, and the fast movement had greater number of sub-

22 movements than the moderate movement $(p s<.05)$. In addition, the visual effect was

23 significant only for the slow movement $(\mathrm{Z}=-3.88, p<.001)$, where the number of sub-

24 movements from the vision condition was greater than that from the no-vision condition $(p$ $25<.05)$. 
For the temporally constrained tasks, the ANOVA result showed the significant main

2 effect of movement goal, $F(1.30,24.75)=213.70, p<.001, \eta_{p}{ }^{2}=.92$, where the slow

3 movement had greater numbers of sub-movements than the moderate and fast movements,

4 and the fast movement also had greater number of sub-movements than the moderate

5 movement condition $(p s<.001)$. The effect of visual condition, $F(1,19)=1.19, p=.29, \eta_{p}^{2}$

$6=.06$, and the movement goal by visual condition interaction, $F(1.21,22.89)=3.10, p=.09$,

$7 \quad \eta_{p}^{2}=.14$, did not reach the significant level.

8

Type of Sub-Movement. Figures 4a to 4 h show the proportion of sub-movement type for vision and no-vision as a function of movement goals. Table 1 and Table 2 show the results from the non-parametric tests for each sub-movement type for spatially constrained tasks and temporally constrained tasks, respectively. For primary sub-movements, we found that the fast movement goal led to the least amount for the spatially constrained tasks and the moderate movement goal led to the most amount for the temporally constrained tasks. Additionally, the no-vision conditions of the slow movement goal had significantly greater number of primary-only sub-movements than the vision conditions of the slow movement goal for both the spatially and temporally constrained tasks.

For pre-peak sub-movements, we observed an increase of sub-movements for slower conditions (for both spatially and temporally constrained tasks). In addition, for the spatially constrained task, the vision condition had significantly greater number of the pre-peak submovements than the no-vision condition for the slow movement goal.

For post-peak sub-movements, the analyses revealed increases in sub-movements for the moderate and slow conditions for both spatially and temporally constrained tasks. In addition, 
1 the vision condition led to greater number of the post-peak type of sub-movements than the

2 no-vision condition in the slow movement goals of both spatially constrained tasks and

3 temporally constrained tasks and also in the moderate movement goal of the temporally

4 constrained task.

Finally, for overshooting sub-movements, we found, mainly, that the number of submovements increased as the task requirements induced faster movements. Additionally, the only visual effect was found in the slow movement of the spatially constrained task where vision condition led to less overshooting sub-movements than the no-vision condition. -Insert Figure 4 and Tables 1 and 2 in here-

\section{Exploratory Analyses}

In order to facilitate interpretation, we have reconfigured the occurrence of submovements as a function of the average MT over the tasks, visual conditions, and the movement goals. Figure 5 shows these results. The data reveal that the task type and the space-time task constraints lead to a different sub-movement profile that is minimally influenced by visual information. That is, the presence of each of the 4 sub-movement types is dependent on task type and space-time task requirements that is mediated by the movement 19 times.

\section{Discussion}

The current experiment was set-up to examine the characteristics of sub-movements in both spatially and temporally constrained discrete aiming tasks (e.g., Fitts \& Peterson, 1964; 
1 Schmidt et al., 1979) under vision and no-vision conditions. The experimental conditions

2 provided a wide range of task constraints to examine the prevalence and type of visually

3 induced sub-movements and their effect on movement accuracy. An experimental emphasis

4 was the inclusion of matched no-vision conditions to act as controls in interpreting the role of

5 sub-movements in the visual control of aiming movements.

\section{$7 \quad$ Visual control of aiming performance}

The pattern of sub-movement probabilities in each space-time and visual condition

9 needs to be interpreted in the context of performance in the speed and accuracy of the aiming

10 outcome. In concert with Fitts' law (Fitts, 1954; Fitts \& Peterson, 1964) the difference of the

11 percentage of success between vision and no-vision conditions became larger when target size of the spatially constrained movement task became smaller. In contrast, the difference of the success rate between vision and no-vision conditions of the time-matching tasks diminished over increasing movement times. Thus, the vision manipulation had differential effects on the task type with it significantly and predominantly enhancing the spatial matching /time-minimization task of the Fitts' law protocol (Wallace \& Newell, 1983). The differential decreasing rate of the success for the spatial tasks between the vision and no-vision conditions reflects the known importance of visual information in the spatial aiming tasks. Providing a visual cue (e.g., size or location of target) and concurrent feedback (e.g., knowing where the limb is during aiming) facilitates the movement accuracy, especially when the requirements of accuracy are more stringent (Crossman \& Goodeve, 1983; Meyer et al., 1990; Newell, Carlton, \& Kim, 1994; Wallace \& Newell, 1983; Zelaznik et al., 2008).

23 The higher success rate of the vision condition at the slow movement goal of ID8 was accompanied with longer movement times and smaller spatial errors (see Figure 2). These 
1 results indicated a possible error correction mechanism of vision that was involved in the

2 execution of the spatial aiming tasks at the slow movement goal.

3 For the timing task, although there was a general direction of longer movement times

4 and smaller spatial errors for the vision condition than the no-vision condition over all

5 movement goals, the only significantly higher success rate between the visual conditions was

6 observed in the no-vision condition of the fast movement goal. The availability of vision

7 seems to have a negative effect on the success rate in the fast movement goal of the

8 temporally constrained (time-matching) tasks. This provided evidence of non-essential role of

9 vision in performing a timing task. It is important to highlight that such a decrease in success

10 rate led to less spatial error in temporally constrained conditions - as the task is known, a

11 "trade-off" occurred. Indeed, the presence of a target with the instruction to finish the

12 movement as close as possible to the target clearly induced spatial constraints on the task -

13 despite the emphasis on the temporal requirements.

\section{Sub-movement characteristics and vision}

16 The traditional interpretation of sub-movement holds that if the primary sub-movement

17 misses, or is anticipated to miss the target, a second or even more sub-movements are

18 performed (Elliott et al., 2001), resulting in a longer movement time. Furthermore, the

19 number of corrective sub-movement increases when the requirements of the target are

20 stringent (Crossman \& Goodeve, 1983; Meyer et al., 1990; Milner \& Ijaz, 1990; Novak,

21 Miller, \& Houk, 2002). Our findings show that there are different probability patterns for

each kind of sub-movement as a function of the task space-time conditions that were

differentially influenced by the presence or unavailability of vision.

The number of sub-movements increased over the longer movement times from about

25 the moderate movement goal of $500 \mathrm{~ms}$ where the trajectories of over $50 \%$ of the trials 
1 showed only the primary sub-movement (Table 2 and Figure $4 \mathrm{~b}$ ). The $2 \mathrm{~mm}$ target size of

2 ID8 (slow movement goal) elicited a long movement time of about $1 \mathrm{~s}$ in the vision condition

3 (Figure $2 \mathrm{c}$ ) and about $80 \%$ of these trials exhibited the post-peak sub-movement (Figure $4 \mathrm{e}$ )

4 that is consistent with the error correction interpretation (Elliott et al., 2001; Milner, 1992).

5 The no-vision condition of ID8 (slow movement goal) had considerably shorter movement

6 time (Figure 2c) and significantly fewer number of sub-movements than those in its vision

7 counterpart (Figure 3a). In fact, there was about $40 \%$ trials of the no-vision condition of ID8

8 (slow movement goal) that had only primary sub-movements (Figure 4a) and another 40\%

9 trials had overshooting sub-movements (Figure 4g). The increased number of sub-movements

10 in the vision condition may be attributed to the increased movement time. Without visual

11 guidance and feedback, the sub-movements observed in the ID8 no-vision condition do not

12 seem to reflect error correction.

13 The fast and moderate movement goals of the spatially and temporally constrained tasks

14 produced average movement times of under $600 \mathrm{~ms}$ (Figure $2 \mathrm{c}, 2 \mathrm{~d}$ ). The trajectory profiles of

15 these tasks produced on average less than 2 sub-movements per trial (Figure 3). The

16 proportion of primary-only sub-movement peaked at the moderate movement goal of about

17 500ms (c.f., Milner, 1992) (Figure 4b) and the proportion of the overshooting sub-movement

18 decreased from $300 \mathrm{~ms}$ (fast movement goal) to $500 \mathrm{~ms}$ (moderate movement goal) in

19 movement times (c.f., Fradet et al., 2008) (Figure 4h). These sub-movement characteristics

20 were observed in both the vision and no-vision conditions. Furthermore, there was no visual

21 effects found for the fast (ID2 and 300ms) and the moderate (ID5 and 500ms) ranges for the

22 primary-only as well as the overshooting type of sub-movement (Table 1 and Table 2, effect

23 of condition).

24 The target size of ID5 (moderate) is about 13\% of ID2 (fast) and the movement times for

25 both visual conditions were significantly longer in ID5 than those in ID2, yet not only were 
1 there significantly more sub-movements per trial in ID2 than ID5, but ID2 also had

2 consistently additional overshooting sub-movement per trial (Table 1 and Figure 4g). A

3 similar trend was also observed in the two shorter movement time goals of the temporal task

4 where there was a higher number of sub-movements in the $300 \mathrm{~ms}$ (fast) condition than the

$5500 \mathrm{~ms}$ (moderate) condition and the type of the extra sub-movements were mainly the

6 overshooting (Table 2 and Figure 4). Taken together, the large enough target size that

7 accommodated almost $100 \%$ successful trials, or the short duration movement time of about

$8300 \mathrm{~ms}$ were associated with a predominant overshooting sub-movement that is consistent

9 with the "braking" action of abrupt movement termination (Dounskaia et al., 2005, Fredet et

10 al., 2008; Hsieh et al., 2019) but is unlikely to be visually induced given the associated short

11 movement time duration.

\section{Visual control of sub-movements}

The vision/no-vision conditions of the slow movement of the time-matching task showed no significant difference in the number of sub-movements. Both visual conditions had very high proportions of pre-peak and post-peak type of sub-movements (Figure 4d, 4f)

17 but very low to none in the overshooting or primary-only categories (Figure 4h, 4b). These results are consistent with the interpretation that the sub-movements are used to regulate the

19 movement duration in the prolonged movements where the accuracy requirement on the 20 spatial target is relaxed (Milner \& Ijaz, 1990; Milner, 1992; Wisleder \& Dounskaia, 2007; van der Wel et al., 2009). Across all of the conditions examined here movement time emerged as the most consequential variable in sub-movement profiles, no matter the other task conditions. Collapsing all the experimental conditions, we were able to observe the number of sub-movements and the proportions of each type of sub-movements as a function of movement time (Figure 5). Very short time movements tend to have overshooting sub- 
1 movement and longer time movements have pre-peak and post-peak sub-movements while

2 moderate time movements most likely have only primary sub-movement.

However, as was observed in the slow movement goal of spatially constrained task,

4 there were also significantly more post-peak type of sub-movements observed in the vision condition than the no-vision condition for the slow movement of the temporally constrained task, and significantly more primary-only sub-movement in the no-vision than vision condition (Table 1 and Table 2, effect of condition). This suggests that when vision is available, the visually guided sub-movement may be an adaptation toward the visible target evidenced by the smaller AE of spatial target and longer movement time in the vision than no-vision condition of the slow movement tasks.

The no-vision control conditions across task types and space-time constraints showed for each of the sub-movement types a similar probability profile of occurrence as the respective vision conditions. The similar no-vision control findings in the time-matching task conditions open the interpretation that the sub-movements in this task, unlike those of the time-minimization protocol of Fitts' law, are not necessarily visually induced given that they are essentially the same as in the no-vision conditions. These parallel vision/ no-vision submovement profiles provide further evidence of different control strategies in timeminimization and time-matching discrete movement tasks.

The profile of sub-movements and the effects of vision/no-vision on them and movement outcome accuracy is strongly task dependent on spatial and temporal constraints as well as their interaction. Vision plays the most important role in the success of the spatially constrained task especially when the target size is small whereas the success of the temporally constrained task does not benefit from vision. However, regardless of the effect of vision and the nature of task constraints, the number of sub-movements as well as the characteristics of sub-movements are directly related to movement times. Very fast 
1 movements tend to have an overshooting sub-movement in addition to the primary sub-

2 movement; movements with moderate speed have higher probability of only primary sub-

3 movement and slow movements usually consist of pre-peak and post-peak sub-movements.

4 Although movement time appears to be the most important variable associated with sub-

5 movement profiles, we could not rule out the possibility of visually induced corrective sub-

6 movement when vision is available.

7

8

9 


\section{Acknowledgements}

2 This research was supported by Ministry of Science and Technology of Taiwan Grant MOST 3 108-2410-H-030-060.

4 
2 Abrams, R. A., \& Pratt, J. (1993). Rapid aimed limb movements: Differential effects of practice on component submovements. Journal of Motor Behavior, 25, 288-298.

4 Carlton, L. G. (1979). Control processes in the production of discrete aiming responses. Journal of Human Movement Studies, 5, 115-124.

6 Carlton, L. G. (1981). Processing visual feedback information for movement control. Journal 7 of Experimental Psychology: Human Perception and Performance, 7, 1019-1030.

8 Carlton, L. G. (1992). Visual processing time and the control of movement. In Advances in psychology, (pp. 3-31). Amsterdam: North-Holland.

Chen, Y., Hoffmann, E. R., \& Goonetilleke, R. S. (2015). Structure of hand/mouse movements. IEEE Transactions on Human-Machine Systems, 45, 790-798.

Chua, R., \& Elliott, D. (1993). Visual regulation of manual aiming. Human Movement Science, 12, 365-401.

Crossman, E. R., \& Goodeve, P. J. (1983). Feedback control of hand-movement and Fitts' law. Quarterly Journal of Experimental Psychology: Human Experimental Psychology,

D’Addio, G., Iuppariello, L., Romano, M., Lullo, F., Pappone, N., \& Cesarelli, M. (2014). Kinematic indexes' reproducibility of horizontal reaching movements. In XIII Mediterranean Conference on Medical and Biological Engineering and Computing

Dounskaia, N., Wisleder, D., \& Johnson, T. (2005). Influence of biomechanical factors on substructure of pointing movements. Experimental Brain Research, 164, 505-516.

25 Dounskaia, N., Fradet, L., Lee, G., Leis, B. C., \& Adler, C. H. (2009). Submovements during 
pointing movements in Parkinson's disease. Experimental Brain Research, 193, 529544.

3 Elliott, D., \& Calvert, R. (1990). The influence of uncertainty and premovement visual information on manual aiming. Canadian Journal of Psychology, 44, 501-11.

Elliott, D., \& Madalena, J. (1987). The influence of premovement visual information on manual aiming. The Quarterly Journal of Experimental Psychology, 39, 541-559.

Elliott, D., Carson, R. G., Goodman, D., \& Chua, R. (1991). Discrete vs. continuous visual control of manual aiming. Human Movement Science, 10, 393-418.

9 Elliott D., Helsen, W. F., \& Chua R. (2001). A century later: Woodworth's (1899) twocomponent model of goal-directed aiming. Psychological Bulletin, 127, 342-357.

Elliott D., Hansen S., Grierson, L. E., Lyons J., Bennett, S. J., \& Hayes S. J. (2010). Goaldirected aiming: two components but multiple processes. Psychological Bulletin, 136, 1023.

Elliott, D., Lyons, J., Hayes, S. J., Burkitt, J. J., Roberts, J. W., Grierson, L. E., ... Bennett, S. J. (2017). The multiple process model of goal-directed reaching revisited. Neuroscience and Biobehavioral Reviews, 72, 95-110.

Fitts P.M. (1954). The information capacity of the human motor system in controlling the amplitude of movement. Journal of Experimental Psychology, 47, 381-391.

Fitts, P. M., \& Peterson, J. R. (1964). Information capacity of discrete motor responses. Journal of Experimental Psychology, 67, 103-112.

Fradet, L., Lee, G., \& Dounskaia, N. (2008). Origins of submovements during pointing movements. Acta Psychologica, 129, 91-100.

Hancock, P. A., \& Newell, K. M. (1985). The movement speed-accuracy relationship in space-time. Motor behaviour: Programming, control, and acquisition (pp. 153-188). Berlin: Springer-Verlag. 
1 Hsieh, T. Y., Pacheco, M. M., \& Newell, K. M. (2015). Entropy of space-time outcome in a movement speed-accuracy task. Human Movement Science, 44, 201-210.

3 Hsieh, T. Y., Liu, Y. T., \& Newell, K. M. (2017). Submovement control processes in discrete aiming as a function of space-time constraints. PLoS One, 12, e0189328.

Hsieh, T. Y., Liu, Y. T., \& Newell, K. M. (2019). Task specificity and the timing of discrete aiming movements. Human Movement Science, 64, 240-251.

Hogan, N. (1984). An organizing principle for a class of voluntary movements. Journal of Neuroscience, 4, 2745-2754.

Keele, S. W. (1968). Movement control in skilled motor performance. Psychological Bulletin, 70, 387-403.

Keele, S. W., \& Posner, M. I. (1968). Processing of visual feedback in rapid movements. Journal of Experimental Psychology, 77, 155-158.

Khan, M. A., \& Franks, I. M. (2003). Online versus offline processing of visual feedback in the production of component submovements. Journal of Motor Behavior, 35, 285-295.

Meyer, D. E., Abrams, R. A., Kornblum, S., Wright, C. E., \& Smith, J. E. K. (1988). Optimality in human motor performance: Ideal control of rapid aimed movements.

Meyer, D. E., Smith, J. E. K., Kornblum, S., Abrams, R. A. \& Wright, C. E. (1990) Speed-

Milner, T. E. (1992). A model for the generation of movements requiring endpoint precision. Neuroscience, 49, 487-496.

Milner, T. E., \& Ijaz, M. M. (1990). The effect of accuracy constraints on three-dimensional movement kinematics. Neuroscience, 35, 365-374. 
1 Morasso, P., \& Ivaldi, F. M. (1982). Trajectory formation and handwriting: A computational model. Biological Cybernetics, 45, 131-142.

3 Moritz, C. T., Barry, B. K., Pascoe, M. A., \& Enoka, R. M. (2005). Discharge rate variability influences the variation in force fluctuations across the working range of a hand muscle. Journal of Neurophysiology, 93, 2449-2459.

Newell, K. M. (1980). The speed-accuracy paradox in movement control: Errors of time and space. In G. E. Stelmach, \& J. Requin (Eds.). Tutorials in motor behavior (pp. 501510). Amsterdam: North-Holland.

Newell, K. M., Carlton, L. G., \& Kim, S. (1994). Time and space-time movement accuracy. Human Performance, 7, 1-21.

Newell, K. M., Carlton, L. G., Carlton, M. J., \& Halbert, J. A. (1980). Velocity as a factor in movement timing accuracy. Journal of Motor Behavior, 12, 47-56.

Newell, K. M., Hoshizaki, L. E. F., Carlton, M. J., \& Halbert, J. A. (1979). Movement time and velocity as determinant of movement timing accuracy. Journal of Motor Behavior, $11,49-58$.

Novak, K. E., Miller, L. E., \& Houk, J. C. (2000). Kinematics of rapid hand movements in a knob-turning task. Experimental Brain Research, 132, 419-433.

Plamondon, R., \& Alimi, A. M. (1997). Speed/accuracy trade-offs in target-directed movements. Behavioral and Brain Sciences, 20, 279-303.

Schmidt, R. A., Zelaznik, H., Hawkins, B., Frank, J. S., \& Quinn, J. T., Jr. (1979). Motoroutput variability: A theory for the accuracy of rapid motor acts. Psychological Review, $86,415-445$.

Shadmehr, R. (2010). Control of movements and temporal discounting of reward. Current Opinion in Neurobiology, 20(6), 726-730.

Vaillancourt, D. E., \& Russell D. M. (2002). Temporal capacity of short-term visuomotor 
memory in continuous force production. Experimental Brain Research, 145, 275-285.

2 van der Wel, R. P. R. D., Sternad, D., \& Rosenbaum, D. A. (2009). Moving the arm at

3 different rates: Slow movements are avoided. Journal of Motor Behavior, 42, 29-36.

4 Wallace, S. A., \& Newell, K. M. (1983). Visual control of discrete aiming movements. Quarterly Journal of Experimental Psychology: Section A: Human Experimental Psychology, 35A, 311-321.

7 Welford, A. T. (1968). Fundamentals of skill. London: Metheun.

8 Welford, A. T., Norris, A. H., \& Shock, N. W. (1969). Speed and accuracy of movement and their changes with age. Acta Psychologica, 30, 3-15.

10 Wisleder D., \& Dounskaia N. (2007). The role of different submovement types during 11 pointing to a target. Experimental Brain Research, 176, 132-149.

12 Woodworth, R. S. (1899). The accuracy of voluntary movement. Psychological Review, 3 (Monograph Suppl.), 1-119.

14 Zelaznik, H. N., Spencer, R. M. C., \& Ivry, R. B. (2008). Behavioral analysis of human movement timing. In S. Grondin (Ed.), Psychology of time (pp. 233-260). Bingley, U.K.:

16 Emerald Group. 
1 Table 1. Statistical results for movement goals and visual conditions for each type of sub2 movement in spatial task.

3

\begin{tabular}{|c|c|c|c|c|c|c|c|c|c|}
\hline & & \multicolumn{5}{|c|}{ Effect of movement goals (MG) } & \multicolumn{3}{|c|}{ Effect of visual conditions } \\
\hline & & $\chi^{2}$ & $d f$ & $p$ & $\mathrm{~W}$ & Post hoc & MG & $Z \&$ post hoc & $p$ \\
\hline \multirow{4}{*}{$\begin{array}{l}\text { Only } \\
\text { Primary }\end{array}$} & \multirow[t]{2}{*}{$\mathrm{V}$} & \multirow[t]{2}{*}{15.36} & \multirow[t]{2}{*}{2} & \multirow[t]{2}{*}{$.001 *$} & \multirow[t]{2}{*}{.38} & \multirow{2}{*}{$\begin{array}{l}\text { slow }>\text { fast, } \\
\text { moderate }>\text { fast }\end{array}$} & fast & -1.13 & .28 \\
\hline & & & & & & & $\bmod$ & -.33 & .74 \\
\hline & \multirow[t]{2}{*}{ NV } & \multirow[t]{2}{*}{17.36} & \multirow[t]{2}{*}{2} & \multirow[t]{2}{*}{$.001 *$} & \multirow[t]{2}{*}{.43} & \multirow{2}{*}{$\begin{array}{l}\text { slow }>\text { fast, } \\
\text { moderate }>\text { fast }\end{array}$} & \multirow[t]{2}{*}{ slow } & -2.79 & \multirow[t]{2}{*}{$.005^{*}$} \\
\hline & & & & & & & & $\mathrm{N}>\mathrm{V}$ & \\
\hline \multirow[t]{4}{*}{ Pre-peak } & \multirow[t]{2}{*}{$\mathrm{V}$} & \multirow[t]{2}{*}{22.13} & \multirow[t]{2}{*}{2} & \multirow[t]{2}{*}{$.001 *$} & \multirow[t]{2}{*}{.55} & \multirow{2}{*}{$\begin{array}{l}\text { slow }> \\
\text { moderate, } \\
\text { slow }>\text { fast }\end{array}$} & fast & -.54 & .59 \\
\hline & & & & & & & $\bmod$ & -1 & .32 \\
\hline & \multirow[t]{2}{*}{ NV } & \multirow[t]{2}{*}{8.73} & \multirow[t]{2}{*}{2} & \multirow[t]{2}{*}{$.01^{*}$} & \multirow[t]{2}{*}{.21} & \multirow[t]{2}{*}{ slow $>$ fast } & \multirow[t]{2}{*}{ slow } & -2.75 & \multirow[t]{2}{*}{$.006^{*}$} \\
\hline & & & & & & & & $\mathrm{V}>\mathrm{N}$ & \\
\hline \multirow{4}{*}{$\begin{array}{l}\text { Post- } \\
\text { peak }\end{array}$} & \multirow[t]{2}{*}{$\mathrm{V}$} & \multirow[t]{2}{*}{36.20} & 2 & $.001 *$ & .91 & slow $>$ moderate & fast & -1 & .31 \\
\hline & & & & & & $>$ fast & $\bmod$ & -.91 & .37 \\
\hline & NV & 23.63 & 2 & $.001 *$ & .59 & slow $>$ & slow & -3.79 & $.001 *$ \\
\hline & & & & & & moderate, fast & & $\mathrm{V}>\mathrm{N}$ & \\
\hline Over- & $\mathrm{V}$ & 38.00 & 2 & $.001 *$ & .95 & fast $>$ moderate & fast & -.71 & .48 \\
\hline shooting & & & & & & $>$ slow & $\bmod$ & -.24 & .81 \\
\hline & NV & 23.68 & 2 & $.001 *$ & .59 & fast $>$ moderate & slow & -2.97 & $.003 *$ \\
\hline & & & & & & $>$ slow & & $\mathrm{N}>\mathrm{V}$ & \\
\hline
\end{tabular}

$4 * p<.05$

5

6 
1 Table 2. Statistical results for movement goals and visual conditions for each type of sub2 movement in temporal task.

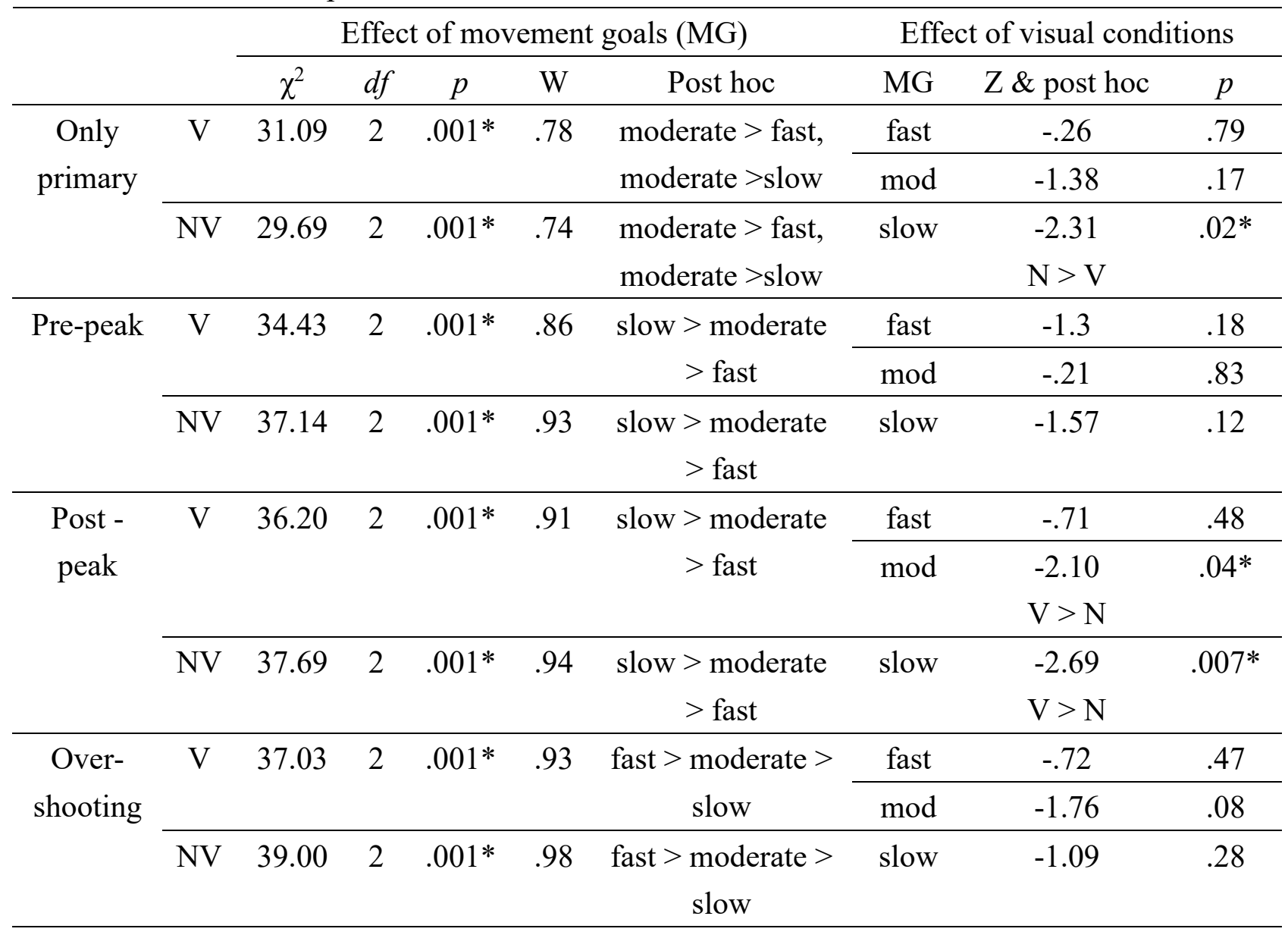

$3 * p<.05$

4

5 
2 1. Examples of different types of sub-movements as defined by Chua and Elliott's (1993) algorithms. The different columns indicate types of sub-movement in displacement (top), velocity (middle) and acceleration (bottom) profiles (from left to right: only primary, prepeak velocity, post-peak velocity and overshooting). The horizontal lines represent the zero level of each kinematic measure and the vertical dash lines indicate the end time for the respective movement. The numbers in parentheses at the bottom of each column indicate the number of the specific type of sub-movement observed in that trial. The primary-only type is always one, four sub-movements of the pre-peak type were found in the second column, two sub-movements of the post-peak type were found in the next

2. Performance of space constrained (left) and time constrained (right) tasks in vison (light symbols) and no-vision (dark symbols) conditions as a function of three movement goals.

4. The proportion of each type of sub-movements in vision and no-vision conditions as a

3. The average number of sub-movements per trial in vison and no-vision conditions as a function of three movement goals for (a) the space constrained tasks and for (b the time constrained tasks. Because there would be at least one sub-movement (primary-only) in a trial, the minimum number of sub-movements per trial is 1 . function of three movement goals for the space constrained tasks (left panels) and for the time constrained tasks (right panels). Panels "a" and "b" are the primary-only submovements; panels "c" and "d" are the pre-peak type of sub-movements; panels "e" and "f" are the pos-peal type of sub-movements; panels "g" and " $h$ " are the overshoot type of sub-movements. 
15 . The occurrence of sub-movements as a function of average movement times of each experimental conditions. Panel "a" shows the average number of sub-movements per trial; panel "b" shows the proportion of primary-only sub-movements, panel "c" shows the proportion of pre-peak type of sub-movements, panel "d" shows the proportion of post-peak type of sub-movements, and panel "e" shows the proportion of overshoot type of sub-movements.

7 


\section{Figure 1}

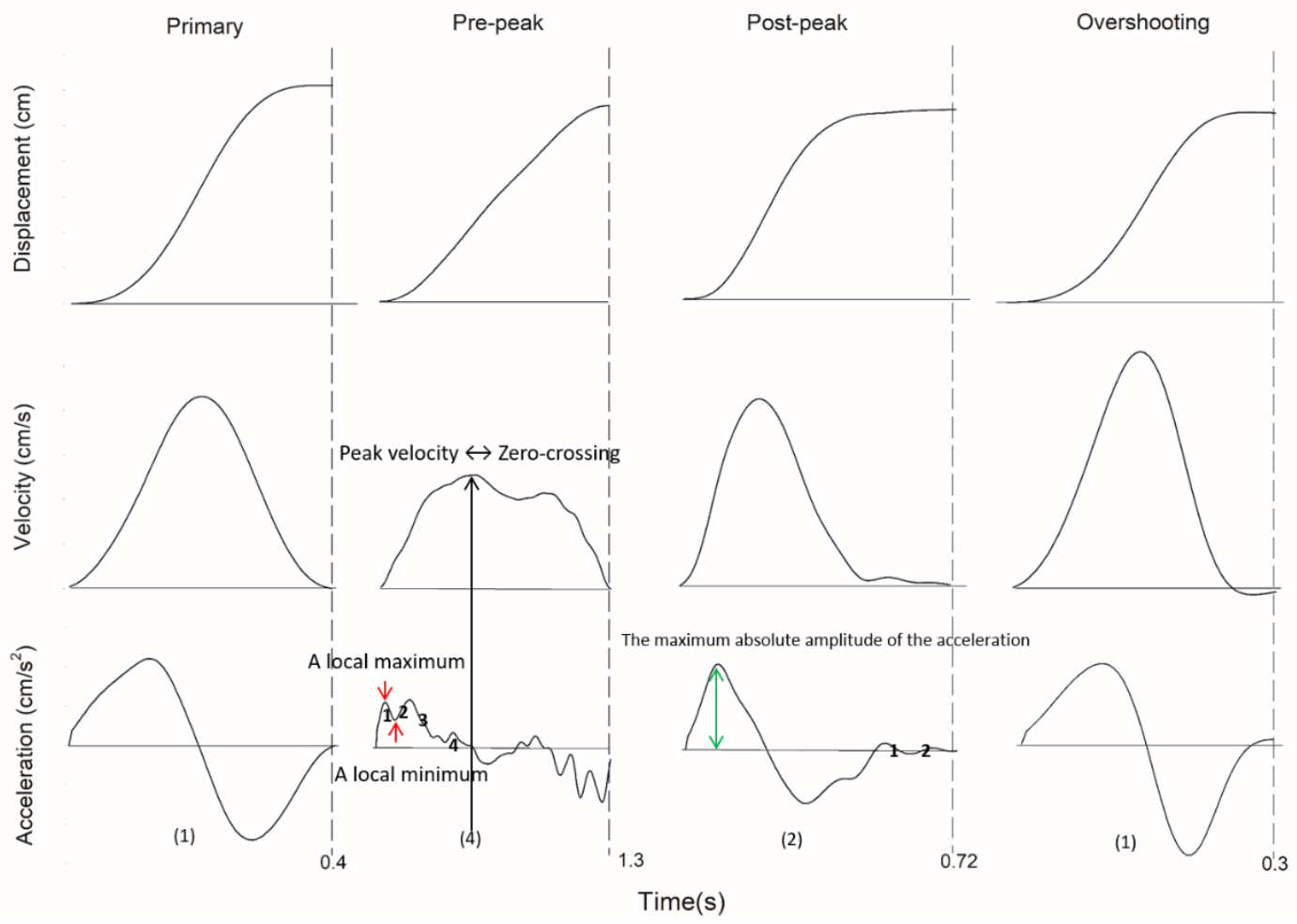

3 
$1 \quad$ Figure 2

Space-Constrained
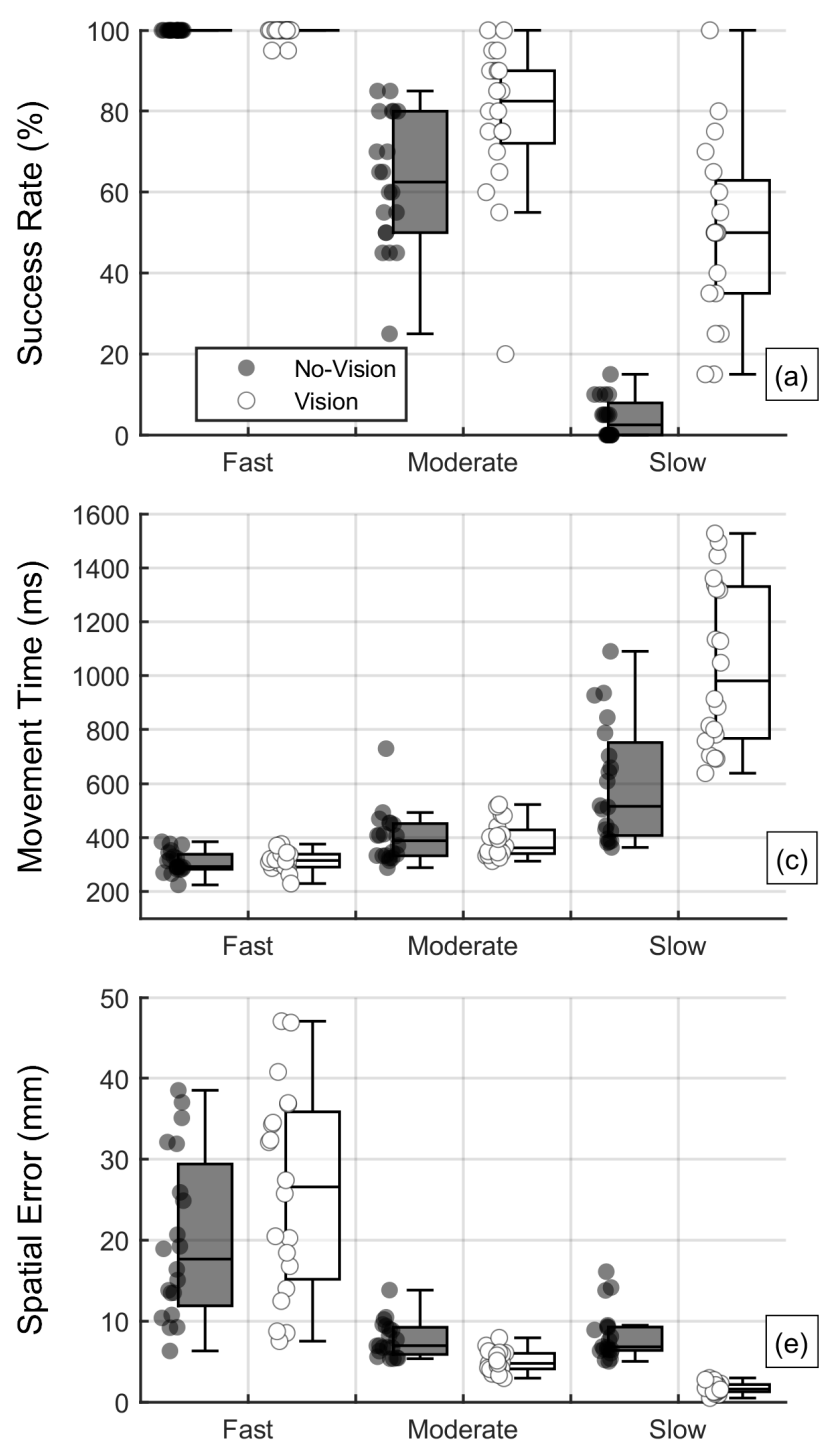

Time-Constrained
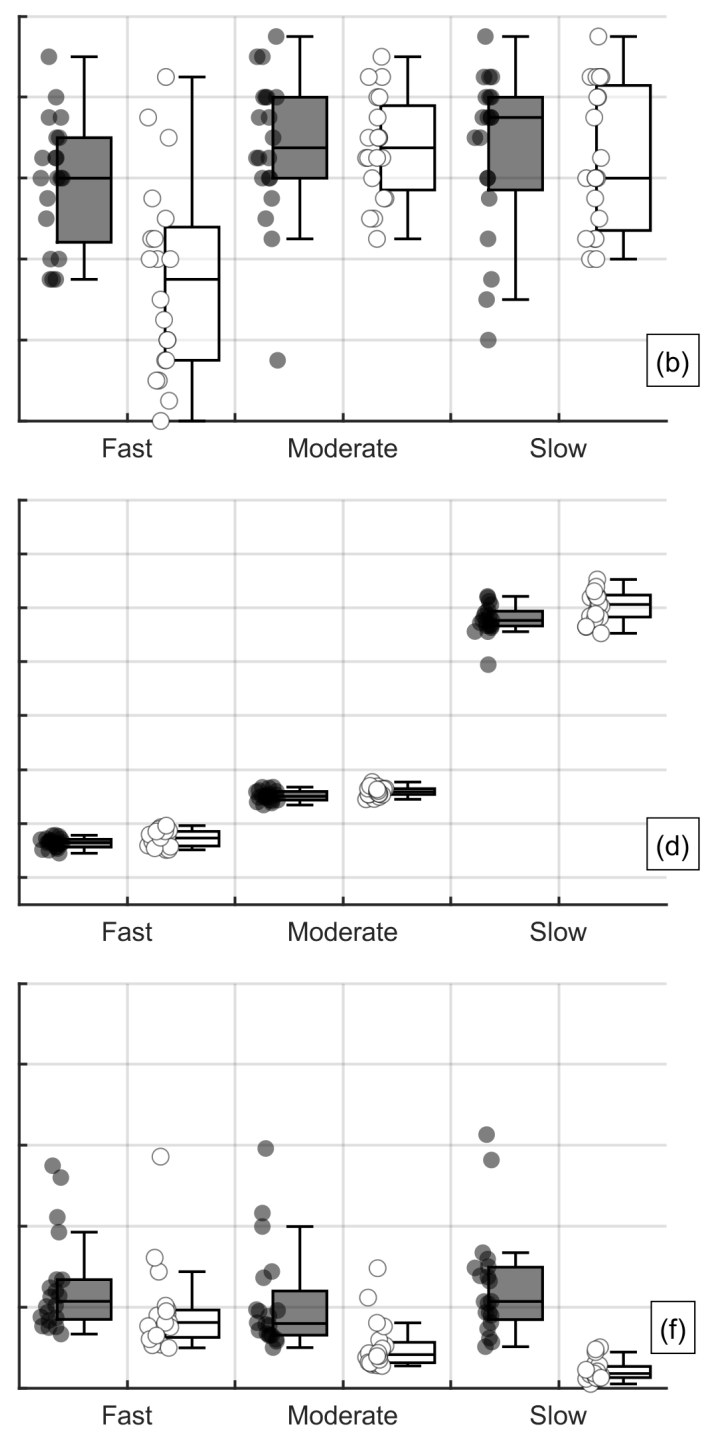
$1 \quad$ Figure 3
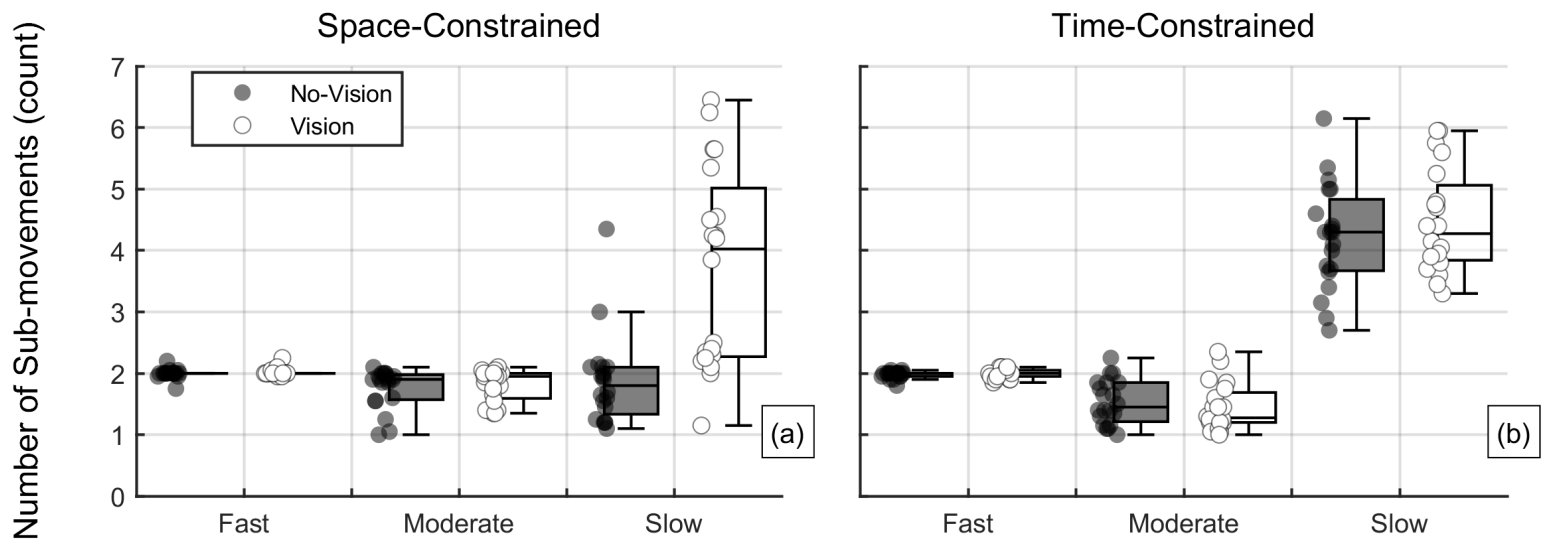

2 
$1 \quad$ Figure 4

Space-Constrained
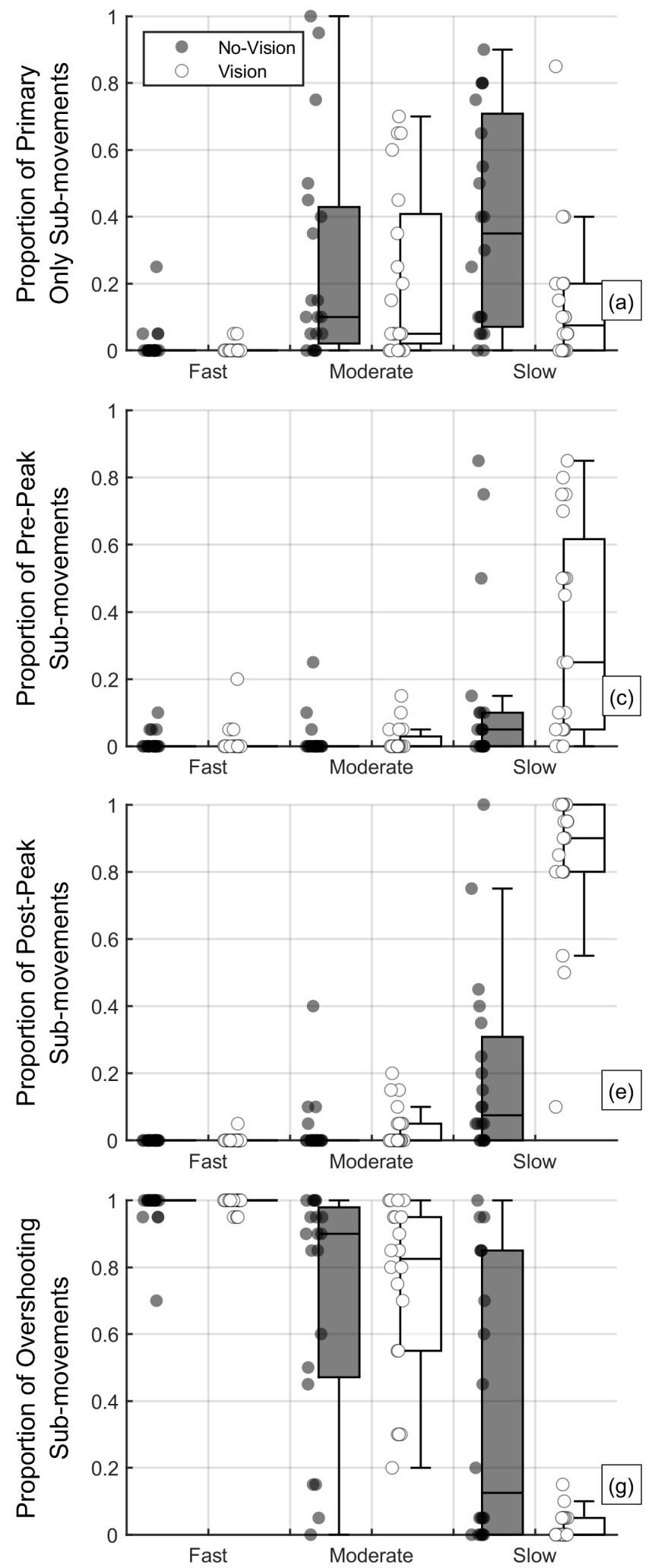

Time-Constrained
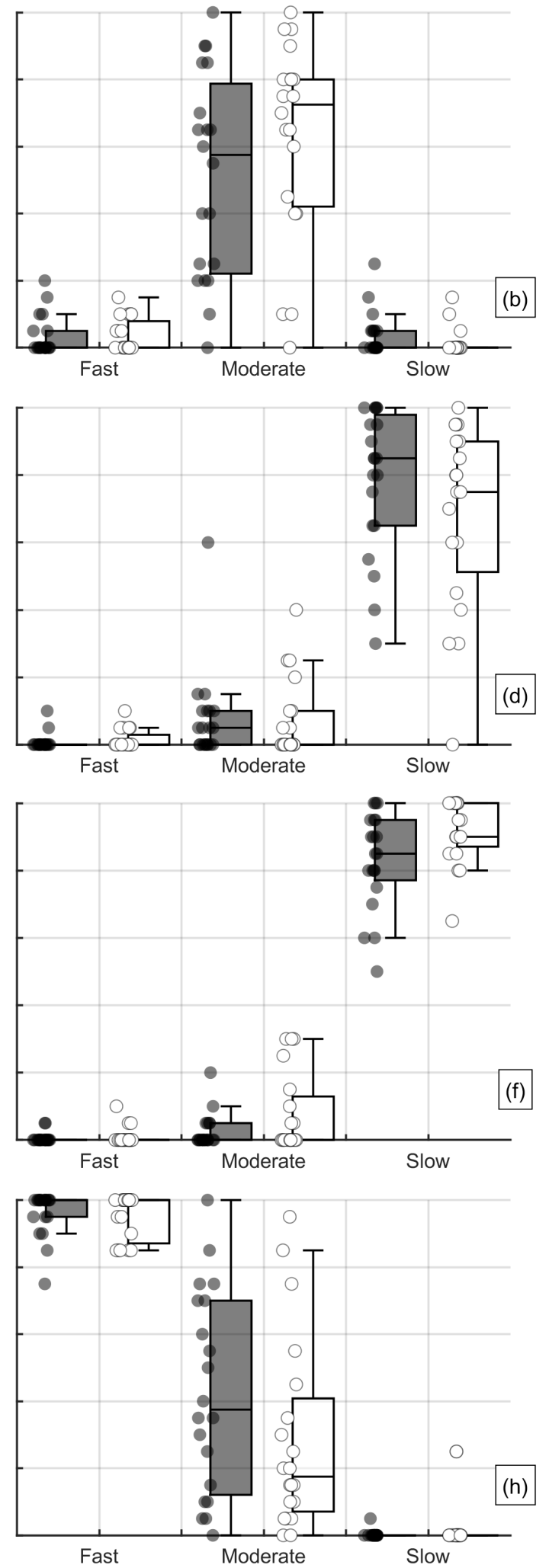
1 Figure 5
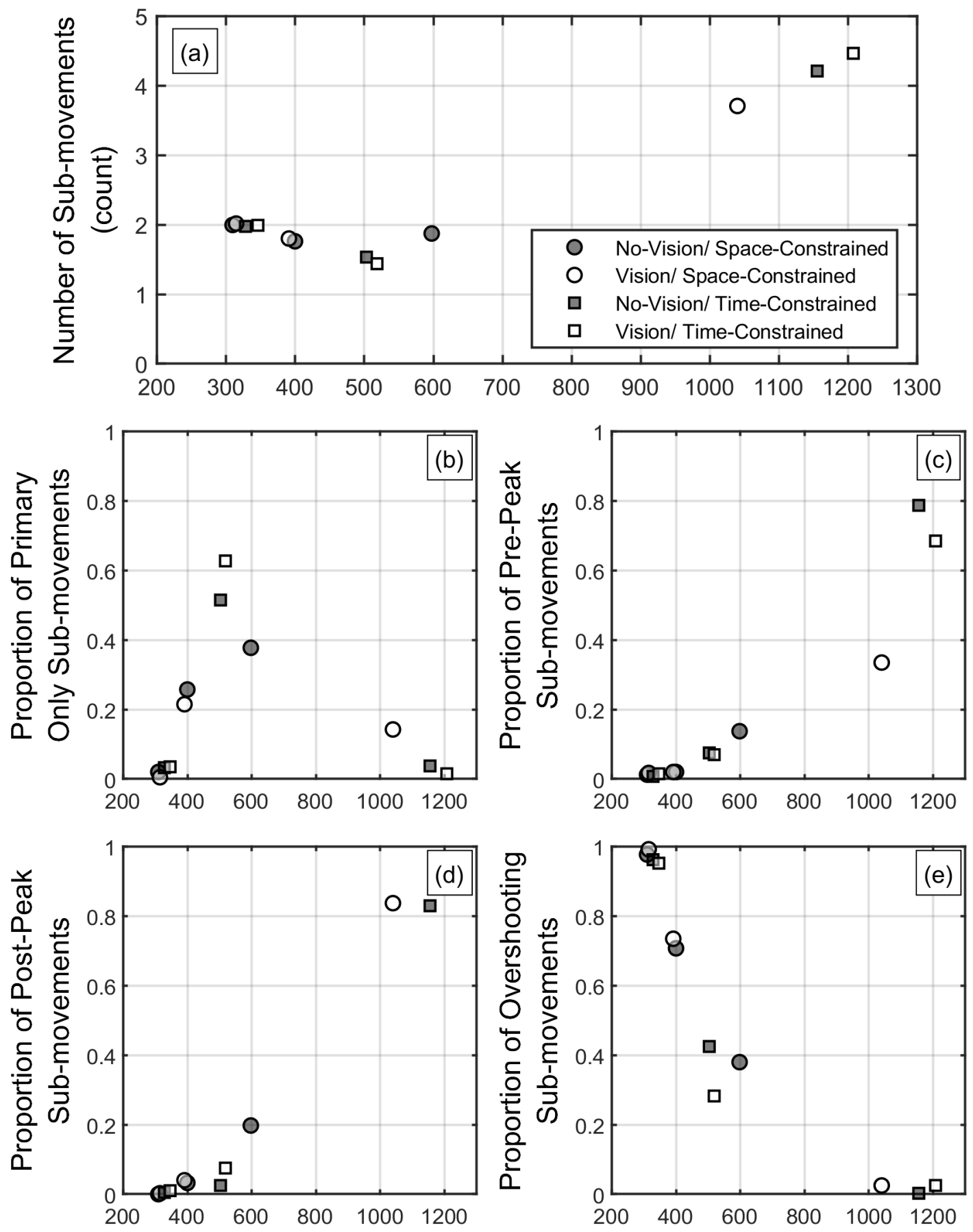

Movement Time (ms) 